\title{
Complex Impedance Matching for Far-Field Acoustic Wireless Power Transfer
}

\author{
Akshayaa YS Pandiyan \\ Department of Electrical \\ and Electronics Engineering \\ Imperial College London \\ London, The United Kingdom \\ a.pandiyan@imperial.ac.uk
}

\author{
Michail E Kiziroglou \\ Department of Electrical \\ and Electronics Engineering \\ Imperial College London \\ London, The United Kingdom \\ m.kiziroglou@imperial.ac.uk
}

\author{
Eric M Yeatman \\ Department of Electrical \\ and Electronics Engineering \\ Imperial College London \\ London, The United Kingdom \\ e.yeatman@imperial.ac.uk
}

\begin{abstract}
In this study, different load matching techniques are analysed to identify the optimum method to deliver power to the receiver for acoustic wireless power transfer systems. Complex impedance matching of the system's transducers provides an advantage to drive the transmitter off-resonance for cases where there is a resonance mismatch between the transducers due to make, defect or ambient conditions. By studying the effect of impedance matching for different frequencies near the resonance frequency, similar power levels can be achieved for a wider bandwidth of frequencies using complex impedance matching. Thus, increased power can be delivered to the receiver by controlling the frequency of the transmitter, which can be exploited for beam steering along the propagation axis when standing waves are prominent between the transducers. A summary of the power experimentally extracted for the different loading techniques presented in this paper demonstrates a $4 \mathrm{kHz}$ increase in system bandwidth and $140 \%$ more power can be delivered by tuning both transducers with complex impedance matching.
\end{abstract}

Index Terms-Wireless Power Transfer, Acoustics, Complex Impedance Matching, Piezoelectric Transducers.

\section{INTRODUCTION}

The application of portable devices for sensing and actuation is proven a necessity recently for security, health and monitoring systems. With a desire to make these devices more feasible, the key concern of powering them has been researched comprehensively including battery technology, energy harvesting (EH) and wireless power transfer (WPT) [1]. Among these, WPT has gained significant attention due to its ease of integration, portability, and ability to recharge isolated nodes. The main WPT techniques under use and research are near field inductive power transfer (IPT), far field RF power transfer and acoustic power transfer (APT), both in near and far field coupling [2]. The transfer efficiency of the IPT systems can reach up to $95 \%$ in the near-field but drops sharply for larger separation distance. On the other hand, acoustic waves can propagate through metal and soil, thereby providing a potential solution for recharging WSN nodes within or behind such materials.

APT systems employ electro-mechanical transducers that can convert mechanical vibrations into electrical energy and vice versa, such as piezoelectric devices. Unlike EH which relies on ambient sources of varying and unpredictable nature, APT uses a power receiver driven by a dedicated (or distributed) remote power transmitter operating at a known frequency. The familiarity of a single known frequency (or a narrow frequency range) allows the design of reactive impedance matching circuitry tailored to the output impedance characteristics of the receiver at that frequency. Furthermore, in piezoelectric EH applications capacitive impedance cancellation requires high inductor values, due to the low frequency operation, which is challenging to integrate in microsystems without substantial additional losses. In contrast, APT systems operate at much higher frequencies (in the ultrasonic range) than piezoelectric EHs, which lowers the required inductance to practical values for matching the impedance of the receiver. Subsequent to the initial power analysis of In-air APT systems in the far-field region by Pandiyan et al. [3], this work studies the improvement of APT efficiency by impedance matching techniques to optimise both the transmitter and the receiver.

Impedance matching in APT systems normally consists of matching the real impedance of the transmitter circuit and electrical load to those of the corresponding transducers. When operated at their resonance frequencies, the transducers have real impedance with no reactive component to tune out. However, there will be circumstances where operation offresonance is necessary or desirable; for example, manufacturing differences or operating differences such as temperature, may cause the transmitter and receiver resonances to differ. Also, reflection between the transducers can prompt standing waves in the far-field region, causing nodes and antinodes that affect the power levels at the receiver. To evade this problem, the transmitter frequency can be detuned to alter the wavelength of the standing waves, so as to place the receiver near a positive antinode. Therefore, in this paper, impedance matching is studied for a range of frequencies near resonance. The piezoelectric system model used for the APT transducers is discussed and experimental results from employing the proposed load matching techniques on an APT evaluation setup for various frequencies are presented.

\section{SySTEM MODEL}

\section{A. Electro-Mechanical Model}

The dynamic behaviour of a piezoelectric transducer can be depicted as an equivalent electrical circuit for a limited range of frequency bordering the resonance, using lumped parameter modelling [4], as shown in Fig. 1. This simplified electrical model consists of an electrical branch interlinked to the mechanical branch through a transformer with $n$ turns ratio representing the piezoelectric coupling vector. The electrical branch consists of the inherent capacitance of the electrodes, $C_{o}$ and the dielectric loss $R_{o}$. However, $R_{o}$ is typically very large and has a negligible effect to the behaviour of the system at the acoustic frequency range [5]-[7]. The mechanical resonance of the transducer can be 


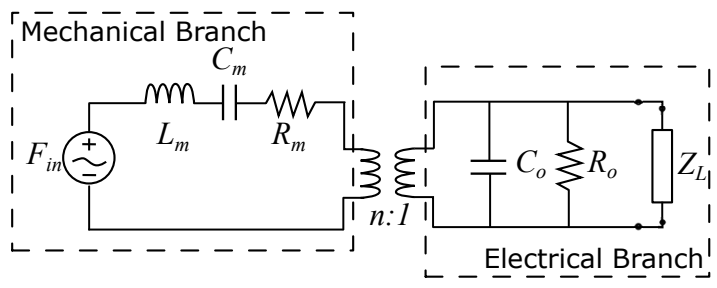

Fig. 1. Lumped model equivalent circuit of a piezoelectric transducer acting as an acoustic power receiver

modelled by the series RLC circuit of the modal mass $\left(L_{m}\right)$, mechanical damping $\left(R_{m}\right)$, and compliance $\left(C_{m}\right)$ of the transducer. Decoupling the electrical and mechanical systems helps analyse the output of the transducer for changing load conditions.

Fig. 1 represents the equivalent network of an APT receiver with a voltage source $F_{\text {in }}$ (analogous to the force of the received sound waves) powering the mechanical branch, and the electrical power from the receiver can be optimised by matching the load impedance $Z_{L}$ with the impedance of the transducer.

\section{B. Impedance Matching}

Maximum power is delivered from an $\mathrm{AC}$ source when the load impedance is the complex conjugate of the source impedance [8], [9]. Fig 2 represents the Norton equivalent of the receiver circuit in Fig. 1. Consider an AC current source from the piezoelectric transducer, $i_{S}(t)=\sqrt{2} I_{S} \sin (\omega t)$ and an internal impedance of $Z_{S}=R_{S}+i X_{S}$. The average power $\left(P_{L}\right)$ delivered to the load impedance $Z_{L}=R_{L}+i X_{L}$ can be calculated as [9]:

$$
P_{L}=\frac{R_{S}^{2}+X_{S}^{2}}{\left(R_{S}+R_{L}\right)^{2}+\left(X_{S}+X_{L}\right)^{2}} I_{S}^{2} R_{L}
$$

When the load is a complex conjugate of the source impedance $Z_{L}=R_{S}-i X_{S}$, then the maximum power delivered to the load is:

$$
P_{L, \max }=\frac{R_{S}^{2}+X_{S}^{2}}{4 R_{S}^{2}} I_{S}^{2} R_{L}
$$

Alternatively, the load and source impedance are matched by using a purely resistive load where $Z_{L}=R_{L}=\sqrt{R_{S}^{2}+X_{S}^{2}}$. This method is favourable for cases that would require large inductance to achieve conjugate matching. As for Eq.2, the maximum power for a purely resistive load can be derived as:

$$
P_{L, \text { max }}^{R}=\frac{R_{S}^{2}+X_{S}^{2}}{2\left(\sqrt{R_{S}^{2}+X_{S}^{2}}+R_{S}\right)} I_{S}^{2} R_{L}
$$

In conclusion, the power delivered to a complex load impedance is higher (Eq.2) when compared to a purely resistive load (Eq.3), especially for transducers operating at

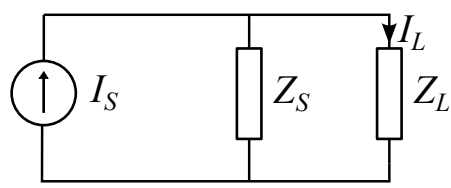

Fig. 2. Norton equivalent of a piezoelectric transducer operating as a receiver.
TABLE I

SPECIFICATIONS OF FUS-40E FROM DATA SHEET

Dual

\begin{tabular}{|c|c|}
\hline Parameters & Value \\
\hline Type & Dual Purpose Transducers \\
\hline Nominal Frequency & $40[\mathrm{kHz}]$ \\
\hline Sensitivity & $\sim 43[\mathrm{~dB}]$ \\
\hline Capacitance & $2[\mathrm{nF}]$ \\
\hline Directionality & $40[\mathrm{Deg}]$ \\
\hline Maximum Input Voltage & $100\left[V_{p-p}\right]$ \\
\hline Outer Diameter & $\phi 16[\mathrm{~mm}]$ \\
\hline
\end{tabular}

lower frequencies or with high reactive impedance. It is also noticeable that for purely resistive loads, the load voltage and current are out-of-phase. Since the reactance of the transducer is a function of frequency, it is essential to evaluate the impedance of the transducer at its operating frequency for selecting the optimal load.

\section{EXPERIMENT RESULTS AND DISCUSSION}

In this work, FUS-40E airborne ultrasonic transducers from Fuji Ceramics were used as both transmitter and receiver for quantifying the power extraction efficiency for resistive and complex loads. They are circular soft ceramic PZT plates glued to a metal diaphragm operating in flexural vibration mode enclosed in a plastic open-type tube. The essential parameters of the transducer are listed in Table I.

\section{A. Frequency Response}

The transducer impedance was measured as a function of frequency in air, using a Precision Component Analyzers 6440B. The measured equivalent resistance and reactance including the resistance of air on the membrane are plotted in Fig. 3 . The inset graph focuses on the impedance trend around $40 \mathrm{kHz}$, which is the nominal frequency of the transducer.

From Fig. 3, the source impedance is purely resistive at $f=39.5 \mathrm{kHz}$, which is the measured resonance frequency of the transducer. At this frequency, the transducer provides the maximum output voltage that can be obtained for a given incident wave, signifying the open-circuit condition. Therefore, using a resistive load to match the impedance at this frequency would be sufficient to deliver maximum power. Considering Fig. 2, the $Z_{S}$ of a piezoelectric transducer can be approximated as a capacitor and resistor in series, which can be matched by a load $Z_{L}$ consisting of an inductor

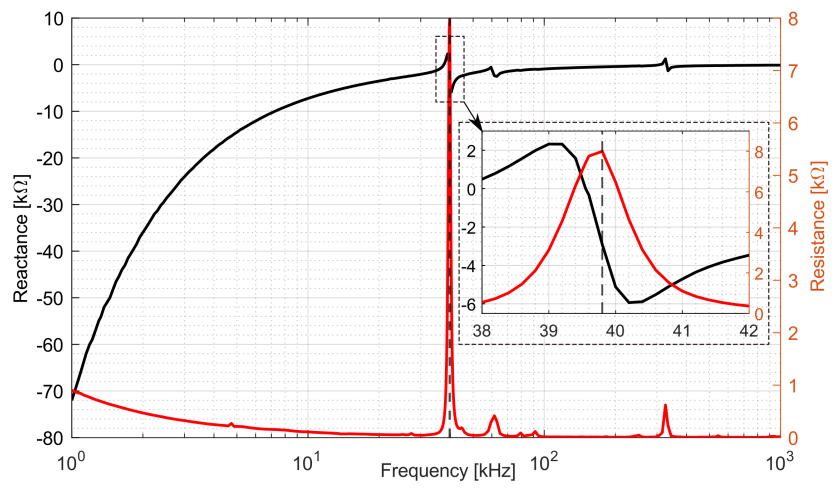

Fig. 3. Equivalent reactance and resistance of FUS-40E piezoelectric transducer measured $25.6^{\circ} \mathrm{C}$ and $20 \%$ dry air. 
TABLE II

Measured Transducer Impedance And CAlCulated VAlues For COMPLEX AND RESISTIVE LOAD MATCHING.

\begin{tabular}{|c|c|c|c|c|c|}
\hline \multirow{3}{*}{$\begin{array}{c}\text { Frequency } \\
\mathbf{k H z}\end{array}$} & \multirow{2}{*}{\multicolumn{2}{|c|}{ Source Impedance }} & \multicolumn{3}{|c|}{ Load Matching } \\
\hline & & & \multirow{2}{*}{$\begin{array}{c}\text { Resistive } \\
R_{L}^{R}[\Omega]\end{array}$} & \multicolumn{2}{|c|}{ Complex } \\
\hline & $\boldsymbol{X}_{S}[\boldsymbol{\Omega}]$ & $R_{S}[\Omega]$ & & $L_{L}[m H]$ & $R_{L}[\Omega]$ \\
\hline 37 & -500 & 190 & 540 & 2 & 190 \\
\hline 37.5 & -100 & 300 & 320 & 0.5 & 300 \\
\hline 38 & 500 & 550 & 750 & $(8.4 n F)$ & 550 \\
\hline 39 & 2300 & 3130 & 4000 & $(1.8 n F)$ & 3130 \\
\hline 39.5 & 0 & 7500 & 7500 & 0 & 7500 \\
\hline 40 & -5100 & 6500 & 8250 & 20 & 6500 \\
\hline 40.4 & -5900 & 3200 & 6700 & 23 & 3200 \\
\hline 41 & -4700 & 1100 & 5000 & 18 & 1100 \\
\hline 41.4 & -4000 & 660 & 4000 & 15 & 660 \\
\hline 42 & -3500 & 360 & 3500 & 13 & 360 \\
\hline
\end{tabular}

and resistor in series. Table II summarises the required load impedance for both resistive and inductive load matching for different transmitter frequencies.

It is also evident from Fig. 3 that from $38 \mathrm{kHz}$ to $39.5 \mathrm{kHz}$, the equivalent reactance of the transducer is positive. This implies that the modal mass dominates the capacitance of the transducers at this frequency range. Consequently, the transducer has an inductive behaviour in this range and a capacitor in series with a resistor is needed for load matching.

\section{B. APT Power Measurements}

To verify the calculated matched impedance values, a FUS$40 \mathrm{E}$ transmitter powered by a STEVAL-IME011V2 highvoltage ultrasonic pulser board from STMicroelectronics is installed on a mounting bracket with precise position control. The pulser board generates a continuous square wave of preprogrammed frequencies with a voltage of $44 V_{p-p}$. As shown in Fig. 4, the receiver is mounted on a rail and can be moved along the $\mathrm{x}$-axis to vary the separation distance between the transducers from 0 to $1 \mathrm{~m}$. This helps to profile the near-, and far-field power transfer. The whole setup is placed in a chamber lined with acoustic foam to reduce reflections and isolate the transducers from external noise. A series network of an inductive (ELC DL07) and a resistive decade box (RS PRO R-100) is connected across the receiver ports to measure the power delivered to the load. The inductance is replaced by a capacitance decade box (TENMA 72-7265) for frequencies between $38 \mathrm{kHz}$ and $39.5 \mathrm{kHz}$.

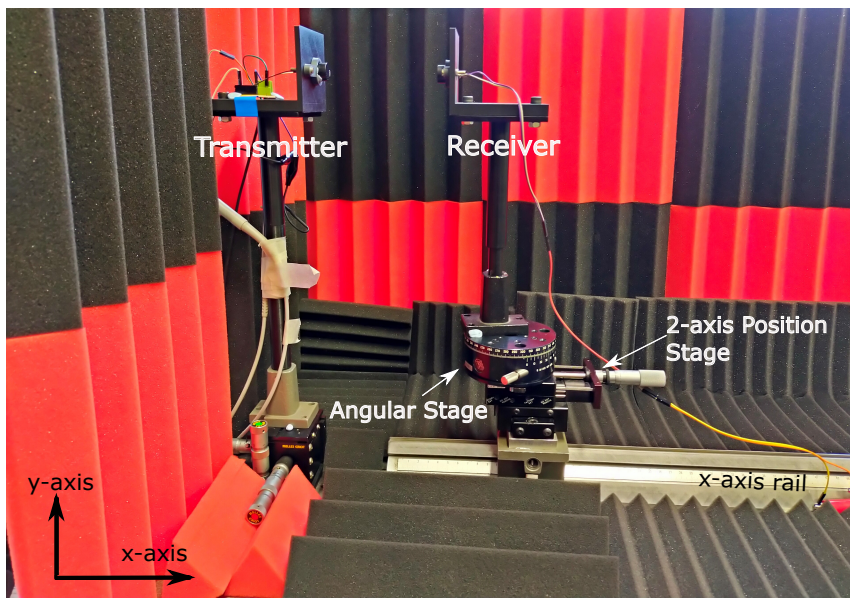

Fig. 4. Experimental setup for profiling complex and resistive impedance matching for far-field acoustic power transfer.
TABLE III

MATCHEd LoAd IMPEDANCE MEASURED From EXPERIMENTS

\begin{tabular}{|r|r|r|r|r|}
\hline \multirow{2}{*}{$\begin{array}{r}\text { Frequency } \\
\boldsymbol{f}[\boldsymbol{k H} \boldsymbol{z}]\end{array}$} & Transmitter & \multicolumn{3}{|c|}{ Receiver } \\
\cline { 3 - 5 } & Inductance & Resistive & \multicolumn{2}{|c|}{ Comple } \\
\cline { 4 - 5 }$[\boldsymbol{m} \boldsymbol{H}]$ & $\boldsymbol{R}_{\boldsymbol{L}}^{\boldsymbol{R}}[\boldsymbol{k} \boldsymbol{\Omega}]$ & $\boldsymbol{L}_{\boldsymbol{L}}[\boldsymbol{m H}]$ & $\boldsymbol{R}_{\boldsymbol{L}}[\boldsymbol{k} \boldsymbol{\Omega}]$ \\
\hline 37 & 0 & 0.5 & 1 & 0.3 \\
\hline 37.5 & 0 & 0.3 & 0.7 & 0.3 \\
\hline 38 & $(8.4 \mathrm{nF})$ & 0.8 & $(8.4 \mathrm{nF})$ & 0.5 \\
\hline 39 & $(2 \mathrm{nF})$ & 4 & $(1.8 \mathrm{nF})$ & 3.5 \\
\hline 39.5 & 0 & 7.5 & 0 & 7.5 \\
\hline 40 & 20 & 10 & 20 & 6.5 \\
\hline 40.5 & 18 & 6 & 23 & 2.4 \\
\hline 41 & 17 & 3.5 & 18 & 1.5 \\
\hline 41.5 & 11 & 3.5 & 15 & 0.5 \\
\hline 42 & 10 & 3 & 13 & 0.3 \\
\hline
\end{tabular}

Fig. 5 shows the output power measured on the load resistor for varying series load impedance, when the receiver was fixed at $10 \mathrm{~cm}$ separation distance from the transmitter. The measurements were taken at an ambient conditions of $25.5( \pm 0.5)^{\circ} \mathrm{C}$ temperature and $20 \%$ R.H. dry air. The solid line represents the power obtained from resistive loading and the dashed lines are reactive loading. The measurements are in agreement with the theoretical calculations. The measured optimum load impedance for both resistive and reactive loading is summarised in Tab. III.

Furthermore, it is evident from Fig. 5 that power emitted by the transmitter decreases as the operating frequency deviates from the nominal frequency. This is because, similar to the receiver, the reactance of the transmitter is significant at non-resonance frequencies, so it is not matched to the signal source. Consequently, an inductor is connected to the transmitter to electrically resonate with the capacitance of the transducer at its operating frequency. By fixing the calculated reactive load matching values for the receiver, the optimal matching inductor for the transmitter is experimentally determined and is summarised in Table III.

Fig. 6 summarises the measured power delivered to the load at the receiver for four different impedance matching techniques: (1) Resistive load matching at receiver (resistive Load) (2) Reactive load matching at transmitter and resistive load matching at receiver ( $\mathrm{T}$ Matched) (3) Reactive

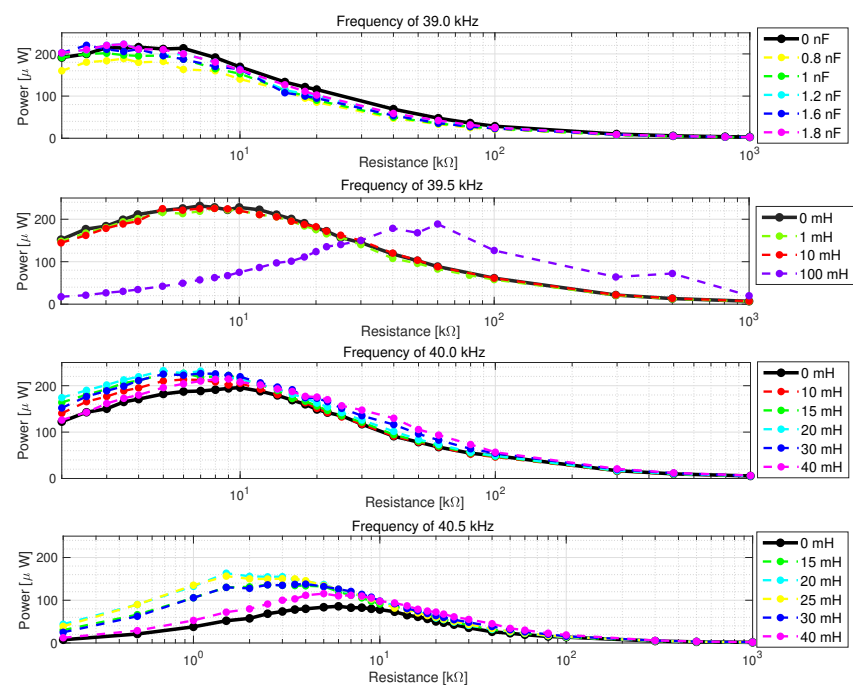

Fig. 5. Resistance sweep of the APT receiver for inductive matched and resistive matched loads to find the maximum power point. 


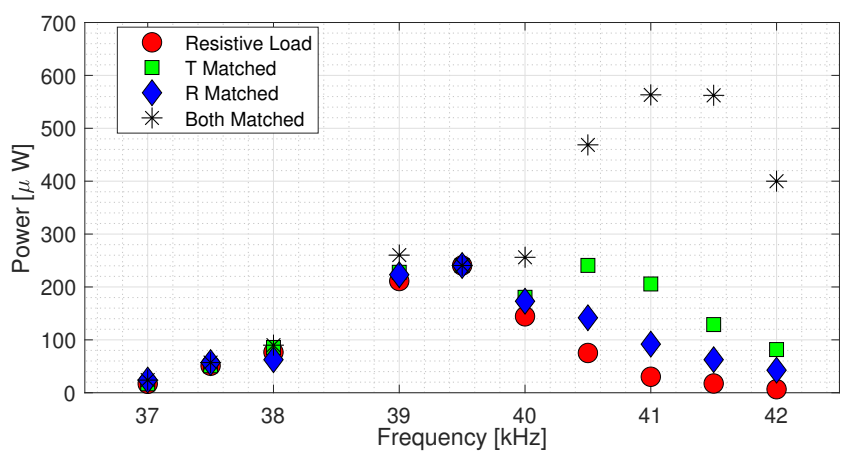

Fig. 6. Experimental maximum power delivered to the load at the receiver for the studied impedance matching techniques.

Load matching at Receiver (R Matched) (4) Reactive load matching at both transmitter and receiver (Both Matched). The APT system yields low power when at the 'Resistive Load' condition at off-resonance where considerable power is dissipated by the transducer reactance as reactive power. When the receiver capacitance is cancelled by an inductor, the peak delivered power increases by up to two times for frequencies with significant reactive impedance.

When the transmitter capacitance is not matched with an inductor $L_{S}$, the source current is $70^{\circ}$ out of phase with transducer voltage. Consequently, more than $65 \%$ of the power delivered to the transmitter is lost as reactive power. By introducing an inductor, the phase difference is reduced to $10^{\circ}$, boosting the delivered power and sound pressure level (SPL). This is apparent from Fig. 6 as the both 'T Matched' and 'Both Matched' consistently have higher power throughput than the other techniques. Furthermore, for operating frequencies off-resonance, the delivered powered is higher than at resonance. For instance, at $41 \mathrm{kHz}$, the received power with and without reactance cancellation is $563 \mu \mathrm{W}$ and $30 \mu W$, boosted by a factor of 17 . However, the low load resistance $(<1 k \Omega)$ (viz. large circuit current) should be considered when designing the conditioning circuit for these cases.

\section{CONCLUSION}

This work demonstrates a comparison between the combinations of reactive and resistive load matching for the transmitter and receiver for APT systems. Experimental results show that at resonance frequency, resistive matching is an acceptable solution as the reactance of the transducer is negligible. However, for operating frequencies higher than resonance, the power delivered to the load substantially increases when both transducers are tuned. For example, the power increased from $240 \mu W$ to $563 \mu W$ when shifting from $39.5 \mathrm{kHz}$ to $41 \mathrm{kHz}$, as both transducers are tuned for optimum power transfer. Additionally, reactance cancellation broadens the operational bandwidth of the APT system by $4 \mathrm{kHz}$ where power greater than $240 \mu \mathrm{W}$ can be achieved. However, the power improvement for operating frequencies less than $39 \mathrm{kHz}$ is negligible, which needs further investigation. Future work will apply the findings to design a selftunable impedance matching circuit for varying frequencies to control the losses due to reflection and standing waves between the transducers.
ACKNOWLEDGMENT

We gratefully acknowledge financial support from the European Commission under the H2020 Marie Sklodowska

Curie ENHANCE project (Grant No: 722496) for this project.

\section{REFERENCES}

[1] M. Safaei, H. A. Sodano, and S. R. Anton, "A review of energy harvesting using piezoelectric materials: state-of-the-art a decade later (2008-2018)," Smart Materials and Structures, vol. 28, no. 11, p. 113001, oct 2019. [Online]. Available: https://doi.org/10.1088/1361$665 \mathrm{x} / \mathrm{ab} 36 \mathrm{e} 4$

[2] M. Roes, J. Duarte, M. Hendrix, and E. Lomonova, "Acoustic energy transfer: A review," Industrial Electronics, IEEE Transactions on, vol. 60, pp. 242-248, 012013.

[3] A. Y. S. Pandiyan, R. L. Rosa, M. E. Kiziroglou, and E. M. Yeatman, "Understanding far field ultrasonic power transmission for automobile sensor networks in free space," in 2019 19th International Conference on Micro and Nanotechnology for Power Generation and Energy Conversion Applications (PowerMEMS), 2019, pp. 1-4.

[4] K. Nakamura, Ultrasonic transducers: Materials and design for sensors, actuators and medical applications. Elsevier, 2012.

[5] N. G. Elvin and A. A. Elvin, "A general equivalent circuit model for piezoelectric generators," Journal of Intelligent Material Systems and Structures, vol. 20, no. 1, pp. 3-9, 2009.

[6] A. Erturk and D. J. Inman, "An experimentally validated bimorph cantilever model for piezoelectric energy harvesting from base excitations," Smart Materials and Structures, vol. 18, no. 2, p. 025009, jan 2009.

[7] A. Erturk, J. M. Renno, and D. J. Inman, "Modeling of piezoelectric energy harvesting from an 1-shaped beam-mass structure with an application to uavs," Journal of Intelligent Material Systems and Structures, vol. 20 , no. 5, pp. 529-544, 2009.

[8] "Introduction to electric circuits." Englewood Cliffs, N.J.,, 1959.

[9] N. Kong, D. S. Ha, A. Erturk, and D. J. Inman, "Resistive impedance matching circuit for piezoelectric energy harvesting," Journal of Intelligent Material Systems and Structures, vol. 21, no. 13, pp. 1293-1302, 2010 . 